\title{
Laparoscopic pancreaticoduodenectomy: a retrospective study of 200 cases and the optimization of the single-center learning curve
}

\author{
Yi-Chen Tang, Qin-Qin Liu, Yong-Gang He, Jing Li, Xiao-Bing Huang \\ Department of Hepatobiliary Surgery, the Second Affiliated Hospital of Army Medical University, Chongqing, China \\ Contributions: (I) Conception and design: YC Tang, J Li, XB Huang; (II) Administrative support: YC Tang, XB Huang, J Li; (III) Provision of study \\ materials or patients: YG He, QQ Liu, YC Tang; (IV) Collection and assembly of data: J Li, YC Tang; (V) Data analysis and interpretation: YG He, \\ QQ Liu; (VI) Manuscript writing: All authors; (VII) Final approval of manuscript: All authors. \\ Correspondence to: Xiao-Bing Huang; Jing Li. Department of Hepatobiliary Surgery, The Second Affiliated Hospital of Army Medical University, No. \\ 183, Xinqiao High Street, Shapingba District, Chongqing 400037, China. Email: 1038915320@qq.com; xqyylj@163.com.
}

Background: Laparoscopic pancreaticoduodenectomy (LPD) is widely used in several centers. This study analyzed the postoperative complications rate curve, possible cause, and solution of LPD and open pancreaticoduodenectomy (OPD).

Methods: Between January 2015 and December 2019, the study included 213 and 204 patients undergoing OPD and LPD, respectively. Postoperative outcomes, complications, and complication risk, along with operation time were analyzed, and the learning curve was determined.

Results: The OPD group (378.7 $\pm 8.98 \mathrm{~min})$ had shorter operation time than the LPD group (402.5 $\pm 7.12 \mathrm{~min})$ $(\mathrm{P}=0.037)$. Blood loss was significantly lower in the LPD group $(389.9 \pm 19.05 \mathrm{~mL})$ than in the OPD group $(530.1 \pm 33.55 \mathrm{~mL})(\mathrm{P}<0.001)$. The incidence of biliary-enteric anastomosis leakage was higher in the LPD group $(2.9 \%)$ than in the OPD group $(0.5 \%)(\mathrm{P}=0.0495)$. The LPD group showed lower lung infection (7.4\% vs. $17.4 \%, \mathrm{P}=0.037)$, incision infection $(1 \%$ vs. $8.5 \%, \mathrm{P}<0.001)$, and anal exhaust time $(3.35 \pm 0.07$ vs. $4.05 \pm 0.07$ days, $\mathrm{P}<0.001)$ than the $\mathrm{OPD}$ group. The biliary-enteric anastomosis leakage was strongly correlated with the pancreatic fistula $(\mathrm{B} / \mathrm{C})(\mathrm{R}=0.6410)$, intraperitoneal infection $(\mathrm{R}=0.6126)$ and ClavienDindo Classification $\geq 3$ ( $\mathrm{R}=0.7403)$. According to the cumulative sum (CUSUM) curve, pancreatic fistula had a negative $\mathrm{K}$ value in 44 cases, biliary-enteric anastomosis leakage had a negative $\mathrm{K}$ value in 46 cases, and Clavien-Dindo Classification $\geq 3$ had a negative $\mathrm{K}$ value in 40 cases. The learning curve for LPD has an inflection point in 86 cases.

Conclusions: LPD is safe and effective for patients with pancreatic cancer, and has a long learning curve and improved postoperative complications in 50 cases. This study's results will help in reducing the complication rates of the first 50 consecutive cases of LPD.

Keywords: Pancreaticoduodenectomy; learning curve; postoperative complications

Submitted Mar 22, 2021. Accepted for publication May 21, 2021.

doi: $10.21037 /$ tcr-21-518

View this article at: https://dx.doi.org/10.21037/tcr-21-518

\section{Introduction}

Pancreaticoduodenectomy (Whipple procedure) has been widely known as an effective treatment for the pancreatic head, especially periampullary carcinoma (1). The traditional open approach has been used for pancreaticoduodenectomy, which includes the resection of the duodenum, thorough lymph node dissection, vascular resection and anastomosis, and reconstruction of the digestive tract. However, patients undergoing the complex operation tend to have a high perioperative complication rate and a long recovery period (2).

Gagner and Pomp first reported the surgical feasibility of laparoscopic pancreaticoduodenectomy (LPD) in 1994, and 
they believed that laparoscopic surgery was more complex than open surgery (3). With the advances of operative techniques, great progress has been observed in LPD in recent years. Studies have also observed the advantages of LPD as follows: lesser blood loss, faster recovery of the gastrointestinal function, and shorter recovery periods compared to the open approach (4-6). Large pancreatic centers have also analyzed the feasibility and learning curve of LPD in large cohort studies $(7,8)$. However, the safety and efficacy of the operation have not been assessed by single centers in large cohort studies (more than 200 cases). Moreover, only little attention has been given to the postoperative complications rate curve, possible cause, and solution of LPD. Therefore, the aim of this retrospective study was to investigate the complications rate curve, possible cause in large cohort studies, and develop a protocol to prevent the severe postoperative complications.

We present the following article in accordance with the STROBE reporting checklist (available at http://dx.doi. org/10.21037/tcr-21-518).

\section{Methods}

\section{Study design}

A total of 417 patients with pancreatic head cancer and periampullary tumor (common bile duct tumor and ampullary tumor), undergoing surgeries at Xinqiao Hospital from January 2015 to December 2019, were included in the study. All the operations were completed by a separate team, guided by the assigned researcher. This separate team had previously completed 3 cases of LPD. Moreover, 213 patients who underwent open pancreaticoduodenectomy (OPD) and 204 patients who underwent LPD were included in this study. LPD and OPD were running at the same time period. The inclusion criteria were: benign and malignant pancreatic tumors located at the head of the pancreas, tumors located at the lower part of the common bile duct and ampulla tumors; had not received gastrointestinal surgery; and no tumor invasion the coeliac trunk, common hepatic artery, and superior mesenteric artery. LPD and OPD had the same inclusion criteria. The exclusion criteria were: those who could not tolerate general anesthesia, and arterial invasion and distant metastases were considered contraindications for the operation. In the early stage, simple cases were selected for LPD, and after 60 cases, LPD and OPD had the same inclusion criteria, such as vascular reconstruction of the SMV. The study was conducted in accordance with the Declaration of Helsinki (as revised in 2013). This study had been approved by the institutional review board of Chongqing Xinqiao Hospital (Approval Number: AF/SC08/1.0; Approval Date: 2018.10.16). Each patient had signed the informed consent with regard to the operation and use of data on their status before and after the operation.

All participating surgeons had performed 50 or more pancreatoduodenectomies (open), completed the training programme in LPD. Before the operation, all patients had undergone the necessary clinical and laboratory examinations to assess the primary tumor invasion, vascular invasion, and distant metastasis. Computed tomography was performed routinely. Before the operation, percutaneous transhepatic cholangial drainage was performed on patients with serum bilirubin exceeding $200 \mu \mathrm{mol} / \mathrm{L}$. According a preoperative risk score model to preoperatively predict the risk of postoperative pancreatic fistula (9). Frozen sections were prepared to examine the surgical margins of the common bile duct/pancreas, the pathologic examination to meet the R0 standard.

\section{Eight-step laparoscopic operative technique (a standard process of pancreaticoduodenectomy)}

(I) Exposure and stripping of the pancreatic head: Kocher maneuver and dissociate the pancreatic head completely, exposing the inferior vena cava, right renal vein, and superior mesenteric artery. (II) Exposure of pancreas and gastrectomy: the gastrocolic ligament was opened, the neck of pancreas was cut, gastrectomy was performed using surgical stapler (EGIAUSYND, EGIA60AVM, EGIA60AMT COVIDIEN). (III) Dissection of porta hepatis: dissect the porta hepatis along common hepatic artery, and divide the common hepatic duct (CHD). (IV) Resection of the uncinate process: cutting off the Treitz ligament and the jejunum, converging into the pancreatic head from the superior mesenteric vein and superior mesenteric artery, the identified blood vessels would be cut off until a complete resection is performed. (V) Reconstruction of the pancreaticojejunostomy: ductto-mucosa reconstruction were performed (measure operative time). The 3-0 Prolene (WB558 ETHICON) was used for the full-thickness suture of the pancreas and outer mucosa of the jejunum. The 5-0 PDS II (W9073 ETHICON) was used for the interrupted suture of the pancreatic duct and jejunal mucosa. (VI) Reconstruction of hepaticojejunostomy: the 5-0 polydioxanone (VCP397H ETHICON), 4-0 V-loc (VLOCL0803 COVIDIEN), and 

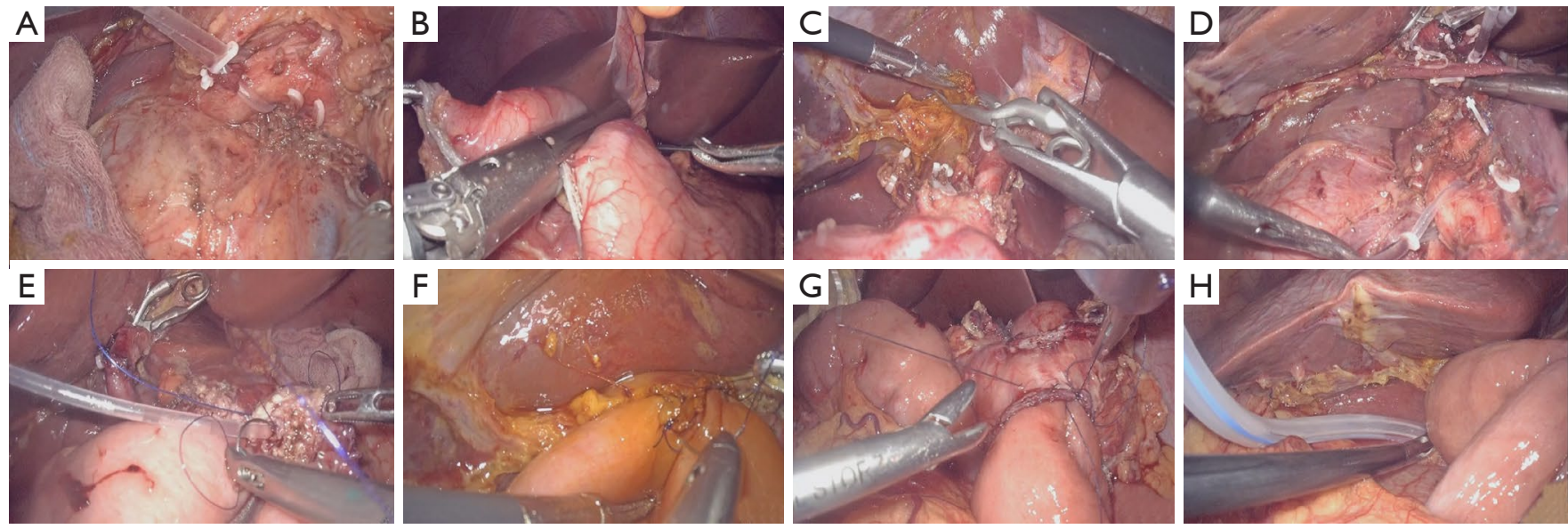

Figure 1 Eight-step laparoscopic operative technique. (A) Exposure and stripping of the pancreatic head; (B) exposure of pancreas and gastrectomy; (C) dissection of porta hepatis; (D) resection of the uncinate process; (E) reconstruction of the pancreaticojejunostomy; (F) reconstruction of hepaticojejunostomy; $(\mathrm{G})$ reconstruction of gastrojejunostomy; $(\mathrm{H})$ placement of the drainage system and closure of the incision.

5-0 PDS II were chosen for hepaticojejunostomy, several sutures were performed to reduce the tension (measure operative time). (VII) Reconstruction of gastrojejunostomy: use a stapler to perform the gastrojejunostomy anterior to the mesocolon (measure operative time). (VIII) Placement of the drainage system and closure of the incision (Figure 1). The above laparoscopic operative were performed with a 3D laparoscopic system (26605AA, STORZ). Gen11 intelligent energy system (ETHICON) and HARMONIC 1100 Shears (ETHICON) were used for energy sealing and dissecting.

\section{Perioperative care}

The nasogastric tube was pulled out 1-3 days after the operation, whereas the abdominal drainage tube was pulled out 3-6 days after the operation. A continuous injection of somatostatin $(6 \mathrm{mg})$ via a micropump was started $24 \mathrm{~h}$ before the abdominal drainage tube was pulled out. Patients began to eat food 2-5 days after operation, depending on their conditions. Subcutaneous unfractionated or fractioned heparin was used for thromboembolic prophylaxis.

\section{Biostatistics and statistical analysis}

We collected clinicopathological characteristics of patients, operative outcomes (operative time, estimated blood loss, histopathological characteristics) and postoperative complications [pancreatic fistulas were diagnosed and classified (9)].

All continuous data were presented as median and mean \pm standard deviation (SD), and frequencies were presented when appropriate for the type of data. The mean values of the continuous variables were compared with a twotailed Student's $t$-test. Nonparametric statistical tests were used if the variables did not follow a normal distribution. Categorical variables were presented as numbers and percentages and were compared using Pearson's $\chi^{2}$ test or Fisher's exact test for contingency tables. For all analyses, a $\mathrm{P}$ value $<0.05$ was considered statistically significant. Moreover, Spearman's rank correlation coefficients were used to analyze relationships of the operative complication. Statistical analyses were carried out using Statistical Product and Service Solutions (SPSS) version 21.0 software (SPSS Inc., IBM, Armonk, NY, USA). The learning curve for LPD and its operative complication rate were evaluated using cumulative sum (CUSUM). CUSUM was conducted using the Intercooled Stata 13.0 statistical software package. GraphPad (7.01 GraphPad Software, San Diego, CA, USA) software was used for plotting.

\section{Results}

\section{Patient characteristics}

The study included a total of 417 patients undergoing pancreaticoduodenectomy in our center, and they were 
Table 1 Clinicopathological characteristics of patients $(\mathrm{n}=417)$

\begin{tabular}{|c|c|c|c|}
\hline Characteristics & OPD $(n=213)$ & LPD $(n=204)$ & $P$ value \\
\hline \multicolumn{4}{|l|}{ Sex, n (\%) } \\
\hline Male & $130(61.0)$ & $120(58.8)$ & \\
\hline Female & $83(38.9)$ & $84(41.2)$ & 0.645 \\
\hline Abdominal surgery in medical history, $\mathrm{n}(\%)$ & $52(24.4)$ & $36(17.9)$ & $0.016^{*}$ \\
\hline \multicolumn{4}{|l|}{ ASA physical status, $\mathrm{n}(\%)$} \\
\hline 1 & $52(24.4)$ & $66(32.4)$ & \\
\hline 2 & $127(59.6)$ & $110(53.9)$ & \\
\hline \multicolumn{4}{|l|}{ Symptoms, n (\%) } \\
\hline Abdominal pain, $\mathrm{n}(\%)$ & $145(68.1)$ & $151(74.0)$ & 0.181 \\
\hline Jaundice, n (\%) & $143(67.1)$ & $127(62.3)$ & 0.297 \\
\hline General weakness, n (\%) & $31(14.6)$ & $20(9.8)$ & 0.139 \\
\hline Tumor size on imaging, mean $\pm \mathrm{SD}, \mathrm{mm}$ & $26.68 \pm 1.34$ & $24.01 \pm 1.01$ & 0.110 \\
\hline Preoperative biliary drainage, $\mathrm{n}(\%)$ & $67(31.5)$ & $77(37.7)$ & 0.177 \\
\hline Endoscopic retrograde biliary drainage, $\mathrm{n}(\%)$ & $13(6.1)$ & $7(3.4)$ & 0.202 \\
\hline Percutaneous transhepatic cholangial drainage, $\mathrm{n}(\%)$ & $54(25.4)$ & $70(34.3)$ & $0.045^{\star}$ \\
\hline High risk for postoperative pancreatic fistula, $\mathrm{n}(\%)$ & $46(21.6)$ & $52(25.5)$ & 0.348 \\
\hline
\end{tabular}

Data are mean \pm SD and $n(\%) .{ }^{*}, P<0.05$ as statistically significant. OPD, open pancreaticoduodenectomy; LPD, laparoscopic pancreaticoduodenectomy; SD, standard deviation; BMI, body mass index; ASA, American Society of Anesthesiology; PDAC, pancreatic ductal adenocarcinoma.

classified into two groups, based on their respective operative methods: OPD group (213 patients) and LPD group (204 patients) (Table 1). The two groups did not differ in age, sex, body mass index (BMI), American Society of Anesthesiology (ASA) physical status, symptom, tumor size on imaging, preoperative biliary drainage, percutaneous transhepatic cholangial drainage, endoscopic retrograde biliary drainage, the number of pancreatic ductal adenocarcinoma (PDAC) patients and high risk for postoperative pancreatic fistula. Fewer patients have a history of abdominal surgery in the LPD group compared to those in the OPD group (17.9\% vs. 22.4\%). Furthermore, more patients with preoperative biliary drainage by percutaneous transhepatic cholangiodrainage (PTCD) were observed in the LPD group than in the OPD group (34.3\% vs. $25.4 \%)$. Differences in the two clinical characteristics were statistically significant $(\mathrm{P}<0.05)$.

\section{Histopathological characteristics and operative outcomes}

In terms of operative outcomes (Table 2), the median operative time of the OPD group was $378.7 \pm 8.98 \mathrm{~min}$, whereas that of the LPD group was $402.5 \pm 7.12 \mathrm{~min}$. Therefore, the OPD group had shorter operative time than the LPD group $(\mathrm{P}=0.037)$. We analyzed the corresponding operative time according to the operative step. In the operative data of the 200 cases, the pancreaticoduodenectomy time in the OPD group was $256.5 \pm 8.31 \mathrm{~min}$, which is shorter than that $(287.4 \pm 6.56 \mathrm{~min})$ in the LPD group $(\mathrm{P}=0.004)$. The pancreaticojejunostomy time in the OPD group was $29.35 \pm 0.41 \mathrm{~min}$, which is significantly shorter than that $(33.81 \pm 0.50 \mathrm{~min})$ in the LPD group $(\mathrm{P}<0.001)$. The 
Table 2 Operative outcomes and histopathological characteristics

\begin{tabular}{|c|c|c|c|}
\hline Clinical characteristics & OPD $(n=213)$ & LPD $(n=204)$ & $P$ value \\
\hline 51-204 cases: operative time, mean $\pm S D$, min & $375.3 \pm 9.31$ & $384.7 \pm 6.42$ & 0.459 \\
\hline Pancreaticoduodenectomy time, mean $\pm S D$, min & $256.5 \pm 8.31$ & $287.4 \pm 6.56$ & $0.004^{*}$ \\
\hline Pancreaticojejunostomy time, mean $\pm \mathrm{SD}$, min & $29.35 \pm 0.41$ & $33.81 \pm 0.50$ & $<0.001^{*}$ \\
\hline Gastrojejunostomy time, mean $\pm \mathrm{SD}$, min & $23.82 \pm 0.41$ & $24.84 \pm 0.40$ & 0.072 \\
\hline Drainage placement and closure time, mean $\pm \mathrm{SD}$, $\min$ & $48.33 \pm 0.50$ & $24.32 \pm 0.70$ & $<0.001^{*}$ \\
\hline Estimated blood loss, mean $\pm \mathrm{SD}, \mathrm{mL}$ & $530.1 \pm 33.55$ & $389.9 \pm 19.05$ & $<0.001^{*}$ \\
\hline Tumor size (mm) & $24.95 \pm 1.30$ & $22.06 \pm 0.88$ & 0.062 \\
\hline Pathological diagnosis, n (\%) & & & 0.094 \\
\hline PDAC & $87(40.8)$ & $60(29.4)$ & \\
\hline Neuroendocrine tumor & $6(2.8)$ & $8(3.9)$ & \\
\hline Intraductal papillary mucinous neoplasm & $13(6.1)$ & $9(4.4)$ & \\
\hline Common bile duct cancer & $53(24.9)$ & $52(25.6)$ & \\
\hline Ampulla of Vater cancer & $31(14.6)$ & $46(22.5)$ & \\
\hline Others & $23(10.8)$ & $29(14.2)$ & \\
\hline Bile duct size $(\mathrm{mm})$ & $13.38 \pm 0.40$ & $14.24 \pm 0.39$ & 0.132 \\
\hline
\end{tabular}

Data are mean $\pm \mathrm{SD}$ and $\mathrm{n}(\%) .{ }^{*}, \mathrm{P}<0.05$ as statistically significant. Others include spindle cell tumors, duodenal cancer, ampulla of Vater adenoma, solid pseudopapillary tumor. OPD, open pancreaticoduodenectomy; LPD, laparoscopic pancreaticoduodenectomy; SD, standard deviation; PDAC, pancreatic ductal adenocarcinoma; LN, lymph node.

drainage placement and closure time in the LPD group was $24.32 \pm 0.70 \mathrm{~min}$, which is shorter than that $(48.33 \pm 0.50 \mathrm{~min})$ in the OPD group $(\mathrm{P}<0.001)$. However, no statistical difference was observed between the two groups with regard to the hepaticojejunostomy time and gastrojejunostomy time. The blood loss in the LPD group was $389.9 \pm 19.05 \mathrm{~mL}$, which is significantly lower than that $(530.1 \pm 33.55 \mathrm{~mL})$ in the OPD group $(\mathrm{P}<0.001)$. Conversion to OPD happened in $14(6.86 \%)$ of the 204 cases undergoing LPD. In terms of histopathological characteristics, no statistical difference was found between the two groups regarding tumor size, pancreatic duct size, pancreas texture, pathological diagnosis, bile duct size, R0 resection rate, lymph node dissection, vascular resection and reconstruction rate, and proportion of malignancy.

\section{Postoperative complications and oncologic outcomes}

After comparing the postoperative complications in the OPD group (213 cases) and LPD group (204 cases), we found that (Table 3) the two groups did not differ in Clavien-Dindo Classification, surgical re-intervention, 
Table 3 Postoperative complications

\begin{tabular}{|c|c|c|c|}
\hline Postoperative complications & OPD $(n=213)$ & LPD $(n=204)$ & $P$ value \\
\hline$<3$ & $196(92.0)$ & $186(91.2)$ & \\
\hline$\geq 3$ & $17(8.0)$ & $18(8.8)$ & 0.757 \\
\hline Surgical re-intervention, n (\%) & $7(3.3)$ & $6(2.9)$ & 0.840 \\
\hline $\mathrm{B}$ & $13(6.1)$ & $10(4.9)$ & \\
\hline C & $3(1.4)$ & $4(2.0)$ & 0.815 \\
\hline Biliary-enteric anastomosis leakage, $\mathrm{n}(\%)$ & $1(0.5)$ & $6(2.9)$ & $0.049^{*}$ \\
\hline Post-pancreatectomy site hemorrhage, n (\%) & $22(10.3)$ & $12(5.9)$ & 0.097 \\
\hline Lung infection, $\mathrm{n}(\%)$ & $37(17.4)$ & $15(7.4)$ & $0.037^{*}$ \\
\hline Incision infection, $\mathrm{n}(\%)$ & $18(8.5)$ & $2(1.0)$ & $<0.001^{*}$ \\
\hline Anal exhaust time, mean $\pm S D$, day & $4.05 \pm 0.07$ & $3.35 \pm 0.07$ & $<0.001^{*}$ \\
\hline Delayed gastric emptying, $\mathrm{n}(\%)$ & $21(9.9)$ & $34(16.7)$ & $0.035^{\star}$ \\
\hline Postoperative hospital stay, mean $\pm \mathrm{SD}$, day & $13.48 \pm 0.56$ & $12.91 \pm 0.65$ & 0.314 \\
\hline
\end{tabular}

Data are mean \pm SD and $n(\%) .{ }^{*}, \mathrm{P}<0.05$ as statistically significant. OPD, open pancreaticoduodenectomy; LPD, laparoscopic pancreaticoduodenectomy; SD, standard deviation.

pancreatic fistula, post-pancreatectomy site hemorrhage, gastrointestinal hemorrhage, intraperitoneal infection, or time of postoperative hospital stay. Among the postoperative complications, the incidence of biliary-enteric anastomosis leakage in the LPD group was $2.9 \%$, which is higher than that $(0.5 \%)$ in the OPD group $(\mathrm{P}=0.0495)$; on the other hand, the incidence of lung infection in the LPD group was $7.4 \%$, which is lower than that (17.4\%) in the OPD group $(\mathrm{P}=0.037)$. Incision infection was found in only $2(1 \%)$ patients undergoing LPD and $18(8.5 \%)$ patients undergoing OPD, therefore the LPD group had significantly lower incision infection than the OPD group $(\mathrm{P}<0.001)$. Furthermore, significantly earlier anal exhaust was observed in the LPD group than in the OPD group ( $3.35 \pm 0.07$ vs. $4.05 \pm 0.07$ days, $\mathrm{P}<0.001)$. We analyzed the correlation between Clavien-Dindo Classification $\geq 3$ and severe postoperative complications by chi-square test. Biliary-enteric anastomosis leakage and intraperitoneal infection were independent factors leading to severe postoperative complications $(\mathrm{P}<0.001)$ (Table 4).

Interestingly, the delayed gastric emptying was observed in $34(16.7 \%)$ patients of the LPD group, and in 21 (9.9\%) patients of the OPD group, $\mathrm{P}=0.035$. To explain this phenomenon, we also extracted and compared the relevant clinical data [operative time, blood loss, the severity of complications, postoperative pancreatic fistula $(\mathrm{B} / \mathrm{C})$, number of days after the nasogastric tube was pulled out, number of days with postoperative activities, postoperative albumin, postoperative pain score, and procalcitonin] of the 34 patients with delayed gastric emptying in the LPD group and the 170 patients without delayed gastric emptying in the LPD group (Table 5). The grade of complication, according to the Clavien-Dindo Classification $(\mathrm{P}=0.03)$ and postoperative pancreatic fistula $(\mathrm{B} / \mathrm{C})(\mathrm{P}<0.001)$, were higher in the group with delayed gastric emptying than in the control group. We also found that removing the nasogastric tube too early (less than 3 days) after the operation was strongly associated with the delayed gastric emptying [odds ratio (OR): $0.058,95 \%$ confidence interval (CI): $0.024-0.141, \mathrm{P}<0.001]$ and might be the critical factor in delaying gastric emptying.

We analyzed the overall survival of LPD and OPD. We excluded 18 cases in the survival analysis [lost to followup (14 cases), 30-day postoperative deaths (4 cases)]. There 
Table 4 Chi-square test was used to analyze the correlation between Clavien-Dindo Classification $\geq 3$ and severe postoperative complications

\begin{tabular}{lccc}
\hline Postoperative complications & $\begin{array}{c}\text { Clavien-Dindo Classification }<3 \\
(\mathrm{n}=186)\end{array}$ & $\begin{array}{c}\text { Clavien-Dindo Classification } \geq 3 \\
(\mathrm{n}=18)\end{array}$ & $\begin{array}{c}\mathrm{P} \text { value } \\
\text { Pancreatic fistula (B/C), } \mathrm{n}\end{array}$ \\
Post-pancreatectomy site hemorrhage, $\mathrm{n}$ & 11 & 2 & 0.085 \\
Gastrointestinal hemorrhage, $\mathrm{n}$ & 10 & 1 & 0.323 \\
Intraperitoneal infection, $\mathrm{n}$ & 20 & 9 & 0.449 \\
Biliary-enteric anastomosis leakage, $\mathrm{n}(\%)$ & 10 & $5(20.6)$ & $<0.001$ \\
\hline
\end{tabular}

$\mathrm{P}<0.001$ as statistically significant.

Table 5 Bootstrap replications in the univariate analyses of the factors related to clinically relevant postoperative delayed gastric emptying ( $\mathrm{n}=34$ ) following LPD

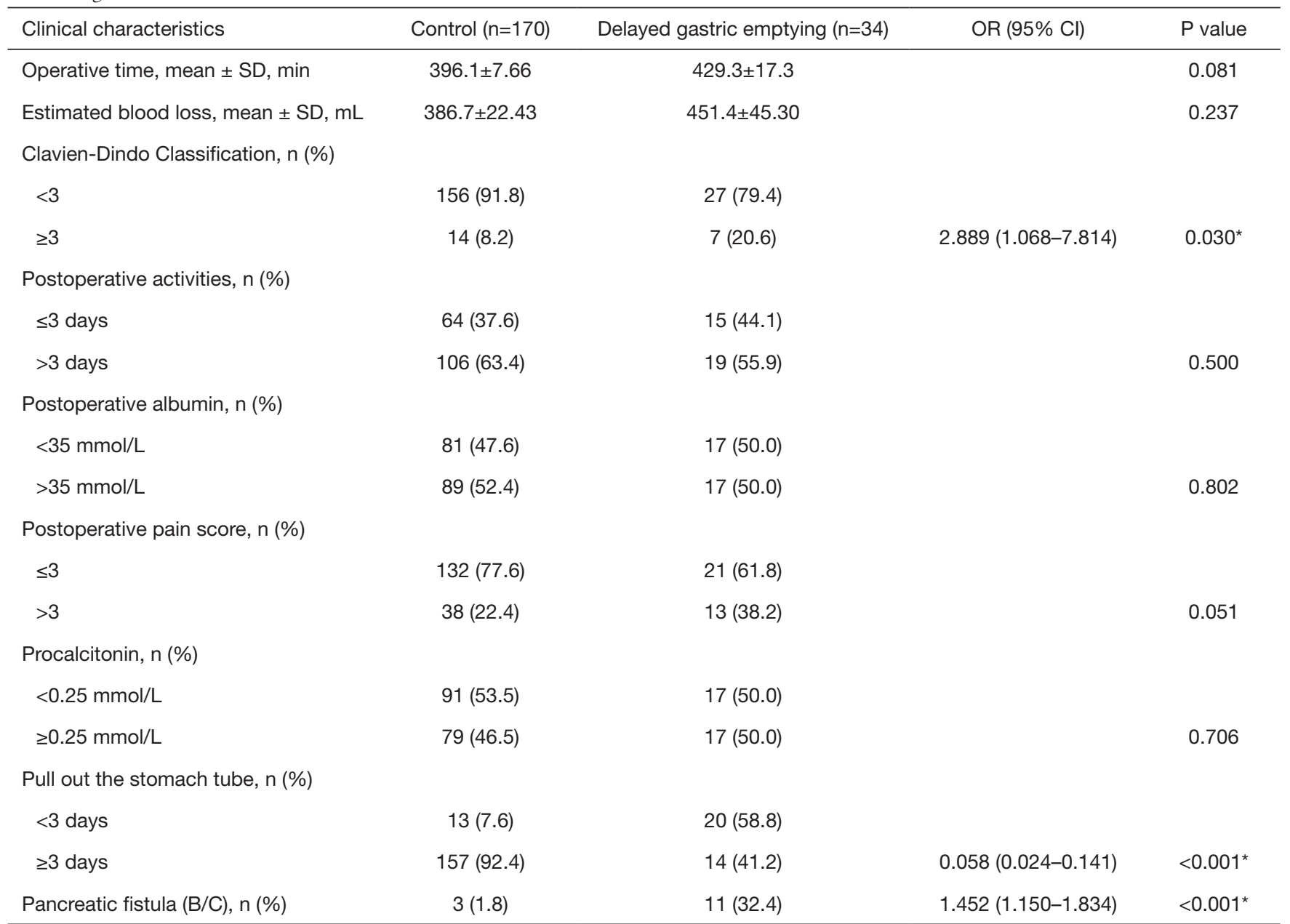

Data are mean \pm SD and $n(\%) .{ }^{*}, \mathrm{P}<0.05$ as statistically significant. LPD, laparoscopic pancreaticoduodenectomy; OR, odds ratio; Cl, confidence interval; SD, standard deviation. 
were no significant difference in overall survival rate between LPD ( $25 \pm 1.6$ months $)$ and OPD $(25 \pm 3.1$ months $)$ $(\mathrm{P}=0.991)$ (Figure 2).

\section{CUSUM analysis of the complication rate and learning curve}

We used the CUSUM to analyze the major postoperative complication rate. The average complication rate in

Kaplan-Meier survival curve of patients with PADC $(n=132)$

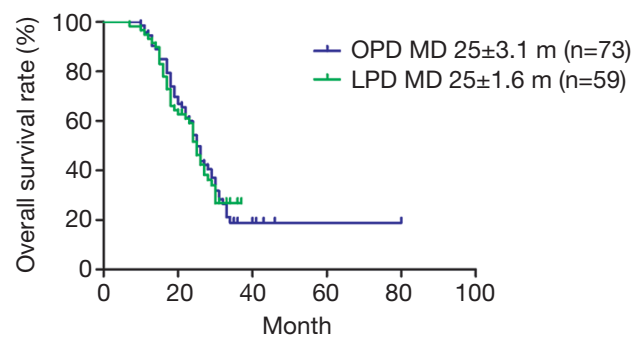

Figure 2 Kaplan-Meier survival curve of patients with PDAC. PDAC, pancreatic ductal adenocarcinoma; OPD, open pancreaticoduodenectomy; LPD, laparoscopic pancreaticoduodenectomy; MD, mean difference.

A

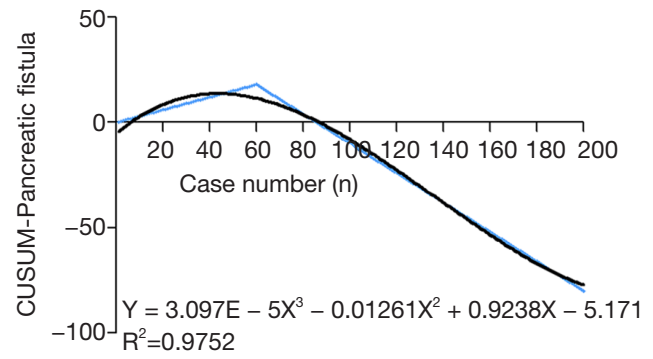

C

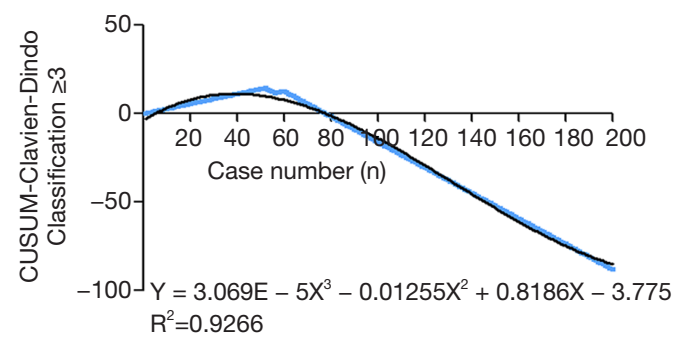

204 patients after LPD was used to judge successes and failures. In the learning curve with a good coefficient of determination, the following were observed: $\mathrm{R}^{2}=0.9752$ for pancreatic fistula with a negative $\mathrm{K}$ value in 44 cases; $R^{2}=0.9949$ for biliary-enteric anastomosis leakage with a negative $\mathrm{K}$ value in 46 cases; and Clavien-Dindo Classification $\geq 3\left(R^{2}=0.9266\right)$ with a negative $K$ value in 40 cases. However, poor-fitting $\left(\mathrm{R}^{2}<0.9\right)$ was observed for post-pancreatectomy site hemorrhage, gastrointestinal hemorrhage, and intraperitoneal infection; therefore, the results were excluded (Figure 3).

CUSUM analysis was performed to determine inflection points for any decrease in the operative time for LPD. We also investigated the risk-adjusted CUSUM plot. The coefficient of determination, $\mathrm{R}^{2}=0.9891$, shows the high fitting. The learning curve comprised three periods based on the changes of consecutive successes or failures (Figure 4).

\section{Discussion}

As LPD techniques advance, LPD or RPD (10-12) have been widely used in more centers. Although LPD has been basically known by the industry for its thoroughness, its safety is still being questioned further. Moreover, the

B

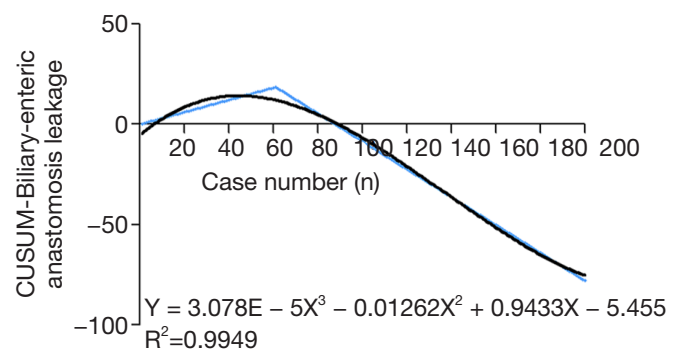

Figure 3 CUSUM analysis the major postoperative complication rate for LPD. (A) CUSUM analysis of pancreatic fistula rate for LPD; (B) CUSUM analysis of biliary-enteric anastomosis leakage rate for LPD; (C) CUSUM analysis of $\geq 3$ of Clavien-Dindo Classification a rate for LPD. The black line is fitted curve. CUSUM, cumulative sum; LPD, laparoscopic pancreaticoduodenectomy. 


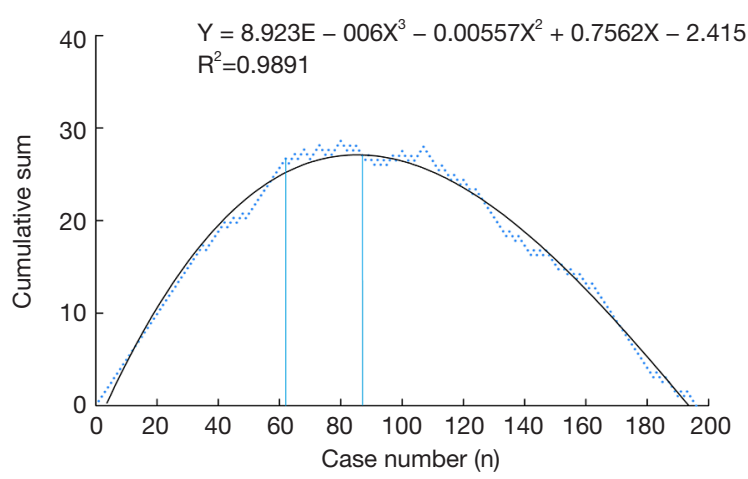

Figure 4 CUSUM analysis of operative time for LPD (the black line is fitted curve). (I) Period 1: study (1-61 cases); (II) period 2: accumulation (62-86 cases). If the slope $\mathrm{K}$ is negative from case 87 , it indicates the start of period 3 ( $87-200$ cases); (III) period 3: the operative time for complete lymph node dissection, vein resection, or pancreatic head tumor resection tends to be lower than the mean operative time. The black line is fitted curve. CUSUM, cumulative sum; LPD, laparoscopic pancreaticoduodenectomy.

correlation between the postoperative complications and prognosis should be further investigated. In our study, the operative time was retrospectively analyzed first. Overall, the operative time for OPD was shorter than that for LPD. This may be associated with the early period of the process of LPD which was time-consuming. Previous studies (13-15) support this finding, but the number of cases increases, the operative time for LPD will not differ significantly with that for OPD after passing the learning curve of LPD. We divided the operation into eight standard processes and found that LPD is more time-consuming than OPD during the pancreas duodenum resection and biliary-intestinal anastomosis. However, LPD is significantly less timeconsuming than OPD during the drainage tube placement and abdominal closure. Therefore, the operative time after passing the learning curve did not differ between LPD and OPD. LPD had significantly less intraoperative blood loss than OPD, there was no clear difference between the two surgical methods during the lymph node dissection. As we have seen, both RPD and LPD have less blood loss and fewer complications compared to OPD. LPD and RPD have similar learning curves and operative time. The author personally think that RPD is better than LPD in gastrointestinal reconstruction, but worse than LPD in surgical field of vision (16). This result may be related to the robot-assisted pancreaticoduodenectomy field of vision. LPD was converted to OPD in 14 patients, of which 10 had severe lymph node metastases in the coeliac trunk and around the superior mesenteric artery, 3 had colon invasion, and 1 had severe intraperitoneal adhesion after the operation.

In terms of postoperative complications, LPD showed lower lung infection, lower incision infection, and faster gastrointestinal exhaust than OPD. These results are consistent with the study of Nassour et al. (17-20). However, no significant difference was found in severe postoperative complication, pancreatic fistula, hemorrhage, postoperative abdominal infection, and time of postoperative hospital stay, but it is worth noting that biliary-enteric anastomosis leakage was found in six patients undergoing LPD. This surgical complication is very rare in OPD and indicates a pancreatic fistula and abdominal infection. Using the chi-square test, we found that the biliary-enteric anastomosis leakage was strongly associated with the severe complications observed after LPD. Continuous polydioxanone suture was applied in five of the six patients who underwent LPD and developed biliary-enteric anastomosis leakage. We suspected that the polydioxanone might damage the bile duct with the thin wall or the infected bile duct so that the duct jejunum anastomotic stoma does not heal, thus resulting in an anastomotic fistula. The 5-0 PDS II was chosen for CJ in the last 154 cases, biliary-enteric anastomosis leakage was no recurrence.

Moreover, we analyzed the cumulative curve of high fitting complications. The severe complications after LPD included pancreatic fistula (6.9\%) and biliary-enteric anastomosis leakage (2.9\%). The severe complication rate was $9 \%$, which is relatively low in the industry (21-23). Therefore, we used our own complication rate as a reference value in the CUSUM of successes. The CUSUM curve of pancreatic fistula had an inflection point in 44 cases, whereas the inflection point of the severe complication curve occurred in 40 cases, which are consistent with the previous studies on LPD reported by Lancet and others (24-26). This means that relatively severe complications may be reduced after 50 cases or more. Many articles on the study of LPD have mentioned that it may be the key for the successful promotion of LPD to encourage surgeons to learn more about, as well as gain more experience in, the early stage of LPD $(27,28)$. Biliary-enteric anastomosis leakage was emphasized in our study since it often indicates biliary fistula, pancreatic fistula, and intestinal leakage. Also a high probability of subsequent abdominal infection, hemorrhage, and pulmonary infection was observed. The incidence of biliary-enteric anastomosis leakage in the LPD 
group significantly higher than the OPD group, and biliaryenteric anastomosis leakage was mostly present in the first 50 cases of LPD. Therefore, we believe that biliaryenteric anastomosis leakage may be the worst complication observed in early stage of LPD, and its prevention could be an important issue to be considered regarding the patient's safety after LPD.

Interestingly, the postoperative gastrointestinal emptying disorder was more noticeable in patients undergoing LPD than in patients undergoing OPD. Moreover, this is in contrast with our previous finding that the time of postoperative intestinal exhaust was shorter for LPD than that for OPD (29). Therefore, we performed a single-factor regression analysis on the conditions of the patients with gastrointestinal emptying disorder after LPD and found that in addition to the severe postoperative complication, the premature removal ( $<2$ days) of the nasogastric tube was an independent factor causing the gastric emptying disorder. It could also be possible that the gastrointestinal decompression was stopped too early for patients undergoing LPD. Furthermore, the 3-4 days after PD may be long enough to pull out the nasogastric tube from patients.

We analyzed the learning curve of LPD. Apart from this, the doctors studied their experience from the first 61 consecutive cases. During this period, the cases with relatively simple medical histories were chosen. Many factors may lead to a prolonged operative time or change of operative method. Moreover, the doctors gained a stable surgical experience from $62-86$ cases. The inflection point occurred after 87 cases, whereas the operative time was shortened. As surgeons are more experienced, performing some of the difficult operations became more feasible, such as pancreatic head cancer operations. More complex operations, such as superior mesenteric vein vascular reconstruction, were performed only after 107 cases. Furthermore, the learning curve of this study was consistent with the findings of van Workum (30). The learning curve of LPD had an inflection point in 80-100 cases.

In summary, after more than 4 years of study on LPD in over 200 cases, we have confirmed the safety and efficacy of LPD. Studies have shown that LPD can achieve effective resection without increasing the risk of complications. However, LPD has a longer learning curve than OPD. The learning curve for LPD has an inflection point in $80-100$ cases. After passing the learning curve, surgeons would summarize a standard process. The severe complication rate after LPD will be significantly reduced after 50 cases. A high risk of biliary-enteric anastomosis leakage may be observed in the early stage of LPD. After the analysis, we found that the best way to prevent biliary-enteric anastomosis leakage is to avoid damaging (such as being cut by a polydioxanone) the bile duct as far as possible; moreover, the surgeon's familiarity with anastomosis is another important factor. We recommend separate training laparoscopic hepaticojejunostomy, in order to be trained in performing the procedure and pass the learning curve before performing LPD. We hope that this study can contribute in the decline of the complication rate in the first 50 consecutive cases of LPD. Further discussions regarding the results of this prospective study will also be included in subsequent research.

\section{Acknowledgments}

The authors wish to thank Dr. Nan You for processing the statistical analysis; and thanks to Enago for the help in editing and revising the manuscript by native English.

Funding: This research is supported by Key R\&D Projects of Chongqing Municipality on Technology Innovation and Application Demonstration Project (project number: cstc2018jscx-mszdX0012).

\section{Footnote}

Reporting Checklist: The authors have completed the STROBE reporting checklist. Available at http://dx.doi. org/10.21037/tcr-21-518

Data Sharing Statement: Available at http://dx.doi. org/10.21037/tcr-21-518

Peer Review File: Available at http://dx.doi.org/10.21037/tcr21-518

Conflicts of Interest: All authors have completed the ICMJE uniform disclosure form (available at http://dx.doi. org/10.21037/tcr-21-518). The authors have no conflicts of interest to declare.

Ethical Statement: The authors are accountable for all aspects of the work in ensuring that questions related to the accuracy or integrity of any part of the work are appropriately investigated and resolved. The study was conducted in accordance with the Declaration of Helsinki (as revised in 2013). This study had been approved by the 
institutional review board of Chongqing Xinqiao Hospital (Approval Number: AF/SC-08/1.0; Approval Date: 2018.10.16). Each patient had signed the informed consent with regard to the operation and use of data on their status before and after the operation.

Open Access Statement: This is an Open Access article distributed in accordance with the Creative Commons Attribution-NonCommercial-NoDerivs 4.0 International License (CC BY-NC-ND 4.0), which permits the noncommercial replication and distribution of the article with the strict proviso that no changes or edits are made and the original work is properly cited (including links to both the formal publication through the relevant DOI and the license). See: https://creativecommons.org/licenses/by-nc-nd/4.0/.

\section{References}

1. Tran TB, Dua MM, Worhunsky DJ, et al. The first decade of laparoscopic pancreaticoduodenectomy in the United States: costs and outcomes using the nationwide inpatient sample. Surg Endosc 2016;30:1778-83.

2. Kamisawa $T$, Wood LD, Itoi $T$, et al. Pancreatic cancer. Lancet 2016;388:73-85.

3. Gagner M, Pomp A. Laparoscopic pylorus-preserving pancreatoduodenectomy. Surg Endosc 1994;8:408-410.

4. Wang S, Shi N, You L, et al. Minimally invasive surgical approach versus open procedure for pancreaticoduodenectomy: a systematic review and metaanalysis. Medicine (Baltimore) 2017;96:e8619.

5. Shin SH, Kim YJ, Song KB, et al. Totally laparoscopic or robot-assisted pancreaticoduodenectomy versus open surgery for periampullary neoplasms: separate systematic reviews and meta-analyses. Surg Endosc 2017;31:3459-74.

6. Torphy RJ, Friedman C, Halpern A, et al. Comparing short-term and oncologic outcomes of minimally invasive versus open pancreaticoduodenectomy across low and high volume centers. Ann Surg 2019;270:1147-55.

7. Shi Y, Jin J, Qiu W, et al. Short-term outcomes after robot-assisted vs open pancreaticoduodenectomy after the learning curve. JAMA Surg 2020;155:389-94.

8. Wang M, Zhu F, Qin R, et al. Which is the best surgical approach for the pancreatic cancer? Classification of pancreatic cancer to guide operative decisions is needed. Ann Surg 2017;265:E81-2.

9. Casadei R, Ricci C, Taffurelli G, et al. Prospective validation of a preoperative risk score model based on pancreatic texture to predict postoperative pancreatic fistula after pancreaticoduodenectomy. Int J Surg 2017;48:189-94.

10. Croome KP, Farnell MB, Que FG, et al. Total laparoscopic pancreaticoduodenectomy for pancreatic ductal adenocarcinoma: oncologic advantages over open approaches? Ann Surg 2014;260:633-8; discussion 638-40.

11. Shi $Y$, Wang W, Qiu $W$, et al. Learning curve from 450 cases of robot-assisted pancreaticoduocectomy in a highvolume pancreatic center: optimization of operative procedure and a retrospective study. Ann Surg 2019. [Epub ahead of print]. doi: 10.1097/SLA.0000000000003664.

12. Wang SE, Shyr BU, Chen SC, et al. Comparison between robotic and open pancreaticoduodenectomy with modified Blumgart pancreaticojejunostomy: a propensity scorematched study. Surgery 2018;164:1162-7.

13. Watkins AA, Kent TS, Gooding WE, et al. Multicenter outcomes of robotic reconstruction during the early learning curve for minimally-invasive pancreaticoduodenectomy. HPB (Oxford) 2018;20:155-65.

14. Lu C, Jin W, Mou YP, et al. Analysis of learning curve for laparoscopic pancreaticoduodenectomy. J Vis Surg 2016;2:145.

15. Han SH, Kang CM, Hwang HK, et al. The Yonsei experience of 104 laparoscopic pancreaticoduodenectomies: a propensity score-matched analysis with open pancreaticoduodenectomy. Surg Endosc 2020;34:1658-64.

16. Marino MV, Podda M, Gomez Ruiz M, et al. Roboticassisted versus open pancreaticoduodenectomy: the results of a case-matched comparison. J Robot Surg 2020;14:493502.

17. Nassour I, Wang SC, Christie A, et al. Minimally invasive versus open pancreaticoduodenectomy: a propensitymatched study from a national cohort of patients. Ann Surg 2018;268:151-7.

18. de Rooij T, Lu MZ, Steen MW, et al. Minimally invasive versus open pancreatoduodenectomy: systematic review and meta-analysis of comparative cohort and registry studies. Ann Surg 2016;264:257-67.

19. Klompmaker S, van Hilst J, Wellner UF, et al. Outcomes after minimally invasive versus open pancreatoduodenectomy: a pan-European propensity score matched study. Ann Surg 2020;271:356-63.

20. Poves I, Burdío F, Morató O, et al. Comparison of perioperative outcomes between laparoscopic and open approach for pancreatoduodenectomy: the PADULAP randomized controlled trial. Ann Surg 2018;268:731-9.

21. Hogg ME, Zenati M, Novak S, et al. Grading of surgeon 
technical performance predicts postoperative pancreatic fistula for pancreaticoduodenectomy independent of patient-related variables. Ann Surg 2016;264:482-91.

22. Palanivelu C, Senthilnathan P, Sabnis SC, et al. Randomized clinical trial of laparoscopic versus open pancreatoduodenectomy for periampullary tumours. Br J Surg 2017;104:1443-50.

23. Roberts KJ, Sutcliffe RP, Marudanayagam R, et al. Scoring system to predict pancreatic fistula after pancreaticoduodenectomy: a UK multicenter study. Ann Surg 2015;261:1191-7.

24. van Hilst J, de Rooij T, Bosscha K, et al. Laparoscopic versus open pancreatoduodenectomy for pancreatic or periampullary tumours (LEOPARD-2): a multicentre, patient-blinded, randomized controlled phase $2 / 3$ trial. Lancet Gastroenterol Hepatol 2019;4:199-207.

25. Adam MA, Thomas S, Youngwirth L, et al. Defining a hospital volume threshold for minimally invasive pancreaticoduodenectomy in the United States. JAMA

Cite this article as: Tang YC, Liu QQ, He YG, Li J, Huang XB. Laparoscopic pancreaticoduodenectomy: a retrospective study of 200 cases and the optimization of the single-center learning curve. Transl Cancer Res 2021;10(7):34363447. doi: $10.21037 /$ tcr-21-518
Surg 2017;152:336-42.

26. Beane JD, Zenati M, Hamad A, et al. Robotic pancreatoduodenectomy with vascular resection: outcomes and learning curve. Surgery 2019;166:8-14.

27. Shyr BU, Chen SC, Shyr YM, et al. Learning curves for robotic pancreatic surgery-from distal pancreatectomy to pancreaticoduodenectomy. Medicine (Baltimore) 2018;97:e13000.

28. Song KB, Kim SC, Lee W, et al. Laparoscopic pancreaticoduodenectomy for periampullary tumors: lessons learned from 500 consecutive patients in a single center. Surg Endosc 2020;34:1343-52.

29. Liu QY, Li L, Xia HT, et al. Risk factors of delayed gastric emptying following pancreaticoduodenectomy. ANZ J Surg 2016;86:69-73.

30. van Workum F, Stenstra MHBC, Berkelmans GHK, et al. Learning curve and associated morbidity of minimally invasive esophagectomy: a retrospective multicenter study. Ann Surg 2019;269:88-94. 